\title{
Impaired Complex-I Mitochondrial Biogenesis in Parkinson Disease Frontal Cortex
}

\author{
Ravindar R. Thomas ${ }^{\mathrm{a}}$, Paula M. Keeney ${ }^{\mathrm{a}}$ and James P. Bennett ${ }^{\mathrm{a}, \mathrm{b}, *}$ \\ ${ }^{a}$ Parkinson's Disease Research Center, Virginia Commonwealth University, Richmond, VA, USA \\ ${ }^{\mathrm{b}}$ Department of Neurology, Virginia Commonwealth University, Richmond, VA, USA
}

\begin{abstract}
Parkinson's disease (PD) can include a progressive frontal lobe $\alpha$-synucleinopathy with disability from cognitive decline and cortico-limbic dysregulation that may arise from bioenergetic impairments. We examined in PD frontal cortex regulation of mitochondrial biogenesis (mitobiogenesis) and its effects on Complex-I. We quantified expression of 33 nuclear genome (nDNA)-encoded and 7 mitochondrial genome (mtDNA)-encoded Complex-I genes, 6 Complex-I assembly factors and multiple mitobiogenesis genes. We related these findings to levels of Complex-I proteins and NADH-driven electron flow in mitochondria from these same specimens reported in earlier studies. We found widespread, decreased expression of nDNA Complex-I genes that correlated in some cases with mitochondrial Complex-I protein levels, and of ACAD9, a Complex-I assembly factor. mtDNA-transcribed Complex-I genes showed $\sim$ constant expression within each PD sample but variable expression across PD samples that correlated with NRF1. Relationships among PGC-1 $\alpha$ and its downstream targets NRF1 and TFAM were very similar in PD and CTL and were related to mitochondrial NADH-driven electron flow. MicroRNA arrays revealed multiple miRNA's regulated $>2$-fold predicted to interact with PGC-1 $\alpha$ or its upstream regulators. Exposure of cultured human neurons to NO, rotenone and TNF-alpha partially reproduced mitobiogenesis down-regulation. In PD frontal cortex mitobiogenesis signaling relationships are maintained but down-regulated, correlate with impaired mitochondrial NADH-driven electron flow and may arise from combinations of nitrosative/oxidative stresses, inflammatory cytokines, altered levels of mitobiogenesis gene-interacting microRNA's, or other unknown mechanisms. Stimulation of mitobiogenesis in PD may inhibit rostral disease progression and appearance of secondary symptoms referable to frontal cortex.
\end{abstract}

Keywords: Parkinson's disease, mitochondrial biogenesis, Complex-I, gene expression, microRNA

\section{INTRODUCTION}

Parkinson's disease (PD) is the second most prevalent neurodegeneration of adults and develops pre-clinically in enteric, olfactory, thoracic sympathetic and medullary neurons before involvement of substantia nigra, where significant neurodegeneration yields the diagnostic bradykinetic movement

\footnotetext{
*Correspondence to: James P. Bennett, Jr., M.D., Ph.D., VCU Parkinson's Disease Center, P.O. Box 980539, Richmond, VA, 23298, USA. Tel.: +1 804828 9556; Fax: +1 804828 6373; E-mail: jpbennett@vcu.edu, http://parkinsons.vcu.edu.
}

disorder late in the pathological progression. Appearance of movement deficits occurs after $>50 \%$ of nigral dopamine neurons have died, by which time Lewy neurite aggregation pathology has already spread rostrally into limbic cortex, followed by progressive involvement of frontal lobes [1-7].

Increasingly successful management of PD motor deficits with drugs and paced electrical brain stimulation in globus pallidus interna or subthalamic nucleus has revealed the progression of "secondary symptoms", such as depression, cortico-limbic dysregulation, cognitive impairment, psychosis and autonomic dysfunction, to be primary disabilities for 
many with PD [8, 9]. Molecular events initiating neurodegeneration ("genesis events") may not be the same as those causing disease progression, both within individuals over time and across populations of PD patients. Such molecular heterogeneity in the setting of close clinical phenotypic similarity of persons with the most prevalent form of sporadic, non-autosomal PD should not be surprising in light of the mutations in seemingly diverse genes responsible for rare, autosomal parkinsonism [10-15].

A major insight into pathogenesis of sporadic PD derived from a gene expression meta-analysis involving individual PD nigral neurons, PD nigral homogenates, preclinical ("incidental Lewy body disease") nigral homogenates, PD putamen homogenates and PD lymphoblasts from living patients. A consistent finding was down regulation of multiple mitochondrial energy metabolism gene sets [16]. As many of these genes were regulated by the master metabolism and mitochondrial biogenesis transcription factor PGC1 $\alpha$ [17], the authors proposed that $\mathrm{PGC} 1 \alpha$ represented a viable target for manipulation to improve PD bioenergetics [16].

Mitochondrial Complex-I is the entry point for transfer of electrons from oxidation of $\mathrm{NADH}$, the major energy carrier from intermediary metabolism. In the same PD postmortem frontal cortex samples used for the present study, we have shown that Complex-I proteins are oxidatively damaged, misassembled and have slowed electron transfer rates from NADH [18]. In these preparations, levels of oxidative damage to Complex-I subunits correlated with reduced NADHdriven electron transfer rates [18]. Levels of multiple Complex-I protein subunits were substantially reduced in the respirasomes of these PD samples, consistent with misassembly, under expression, enhanced degradation, or some combination [19]. To explore further how Complex-I production is regulated in these PD frontal cortex samples, we undertook a comprehensive, qPCR-based examination of expression of Complex-I genes coded for by the nuclear genome (nDNA, 33 out of 38) and mitochondrial genome (mtDNA, 7 out of 7). In addition we examined expression of genes for the 6 known Complex-I assembly factors and several upstream transcription factors involved in mitochondrial biogenesis (mitobiogenesis) [17, 20, 21].

We related the reduced expression of specific nDNA-encoded Complex-I genes to mitochondrial levels of their protein products. We found reduced expression of several mitobiogenesis transcription factors/cofactors, related those levels to mitochondrial $\mathrm{NADH}$-driven electron transfer rates and searched for brain microRNA's that could be involved in regulation of these factors. Finally, to mimic the oxidative-nitrosative stress and inflammatory cytokine environment of PD neurons in situ, we exposed human neurons in culture to DETA-NO, alone or in combination with TNF-alpha or rotenone and assayed the effects of these exposures on mitobiogenesis gene expression.

Overall we found in PD frontal cortex a pervasive downregulation of nuclear-encoded Complex-I gene expression that was associated with decreased expression of mitobiogenesis factors, loss of a recently described Complex-I assembly factor ACAD9 and regulation of some but not all examined Complex-I proteins. Relationships between PGC- $1 \alpha$ and its downstream mitobiogenesis factors NRF1 and TFAM were identical in PD brains compared to CTL and predicted mitochondrial NADH-driven electron transfer rates. These findings suggest that the deficit in $\mathrm{PD}$ is at or above the level of PGC- $1 \alpha$ expression, which in turn is regulating Complex-I mitochondrial electron transfer function. Regulation of expression of the seven mtDNA-encoded Complex-I genes varied across PD samples and was directly related to NRF1. We also found changes in PD brain microRNA's that could alter PGC- $1 \alpha$ expression, as well as downregulation of mitobiogenesis in human neurons elicited by combinations of oxidative/nitrosative stress and cytokine (TNF- $\alpha)$. Thus, mitobiogenesis is impaired in PD frontal cortex with bioenergetic consequences at Complex-I, but the underlying mechanisms are likely multifactorial.

\section{MATERIALS AND METHODS}

Frontal cortex grey matter ribbons from PD and CTL brain samples were retrieved from the University of Virginia Brain Resource Facility where samples had been collected under an IRB-approved protocol. Total RNA including micro RNA's was isolated using Qiagen (Valencia, CA) miRNeasy ${ }^{\circledR}$ kits. BeckmanCoulter Genomics (Morrisville, NC) carried out the miRNA arrays using Agilent Human miRNA Version 3 microarrays. All microarray data was processed using Genespring GX v11 (Agilent Technologies). cDNA preparations were made using iScript (Bio-Rad, Hercules, CA) and random hexamer primers. Multiple cDNA preparations from each brain RNA sample were made at the same time and pooled. In this manner, all qPCR assays could be performed from the same cDNA lots. qPCR primers and multiplex probes were 
designed with Beacon Designer ${ }^{\circledR}$ (Premier Biosoft International, Palo Alto, CA), which did not allow creation of acceptable qPCR primer pairs for 5 of the 38 nDNA-encoded Complex-I genes. qPCR was carried out in a CFX96 thermocycler (Bio-Rad) using BioRad master mixes for either syber green detection for individual genes or a multiplex approach for detection of housekeeping genes. All qPCR experiments on human brain tissue were carried out by the same operator (RRT) who was blinded as to their identity. The primer/probe sequences used are available from the authors (JPB).

Presentation of frontal cortical mitochondrial NADH-driven electron transfer (assayed as superoxide production rate) and mitochondrial levels of ComplexI proteins have previously been reported from our group in Keeney et al. [18] and Arthur et al. [19], respectively. In these earlier studies we used many of the same CTL and PD frontal cortex samples used in the current study. Where overlap existed among sample sources, we combined data for presentation in the current study.

\section{DAN cell culture}

Human Dopaminergic Neuronal Precursor Cells (DAN) were purchased from Clonexpress (Gaithersburg, MD) and cultured according to the supplier's instructions. Briefly, cell pellets were thawed, spun and transferred to a T75 flask with growth medium consisting of DMEM/F12 1:1 (Hyclone,Logan Utah), 5\% fetal bovine serum (Hyclone), Antibiotic/Antimycotic (Invitrogen, Carlsbad, CA), $10 \mathrm{ng} / \mathrm{ml}$ bFGF (Sigma, St Louis, Mo) and $10 \mathrm{ng} / \mathrm{ml}$ GDNF (Sigma). Seven days before experimental treatment, cells were lifted from the flask using Accutase (Invitrogen) and plated in $35 \mathrm{~mm}$ poly lysine coated dishes (MatTek, Ashalnd, MA) with differentiation medium consisting of DMEM/F12 1:1 (Hyclone), 5\% fetal bovine serum (Hyclone), Antibiotic/Antimycotic (Invitrogen), $10 \mathrm{ng} / \mathrm{ml}$ bFGF (Sigma, St Louis, Mo), $10 \mathrm{ng} / \mathrm{ml}$ EGF and $10 \mathrm{uM}$ dibuturyl cAMP. For both growth and differentiation, medium was changed every 3 to 4 days and cells were kept at $37^{\circ} \mathrm{C}$ in a humidified $\mathrm{CO} 2$ incubator.

\section{Treatment of DAN cells and nucleic acid purification}

After seven days of differentiation, culture medium was removed and replaced with the NO generator $100 \mathrm{uM}$ DETA-NO (Sigma), $1 \mathrm{ng} / \mathrm{ml}$ TNF $\alpha$ (Sigma),
$30 \mathrm{nM}$ rotenone or a combination of these in differentiation medium. Culturing continued for seven days with a medium exchange at day three. On day seven cells were lifted with Accutase (Invitrogen), washed, and each pellet sonicated in 350 ul Buffer RLT Plus (Qiagen). DNA and RNA were isolated from independently cultured triplicate samples for each treatment using the AllPrep DNA/RNA Mini Kit (Qiagen) and quantified using a NanoDrop 2000c. 372 ng of RNA from each sample was reverse transcribed to cDNA using iScript kit (Bio-Rad, Hercules, CA).

\section{Graphing and statistics}

GraphPad Prism ${ }^{\circledR}$ (GraphPad Software) was used to construct all graphs. Statistical analyses were carried out using Prism and InStat (GraphPad Software).

\section{RESULTS}

For this study we used frontal cortex samples (8 PD, $8 \mathrm{CTL}$ ) from many of the same cases used in our earlier studies $[18,19,22]$. In these studies we showed that PD cortex gradient-purified mitochondria had reduced NADH-driven electron flow with increased oxidative damage to immunocaptured Complex-I proteins [18]. The loss of NADH-driven electron flow correlated with degree of oxidative damage [18]. Complex-I was misassembled, with reduced levels of individual Complex-I proteins normalized to mitochondrial mass [19].

In the present study we undertook a more extensive examination of Complex-I regulation in these PD frontal cortex samples. Figure 1 shows the results of qPCR analyses for 33 out of the 38 known nDNA-encoded Complex-I genes ("NDU"), where their expression is presented as expression relative to the averaged value for the $8 \mathrm{CTL}$ samples. When results from all NDU genes were grouped together, there was a highly significant $54 \%$ reduction in expression in the PD samples, with a biphasic distribution relative to CTL (Fig. 1).

Expression of individual NDU genes varied in PD cortex relative to mean control values (Fig. 2). Sixteen showed greater than two-fold decreases $(\% \mathrm{CTL}$ $<50 \%$ ).

Complex-I assembly is a complex process that depends on the availability of assembly factors. Figure 3 shows that of the six known Complex-I assembly factors examined, expression of the recently described ACAD9 [23] was decreased to $<20 \%$ CTL 


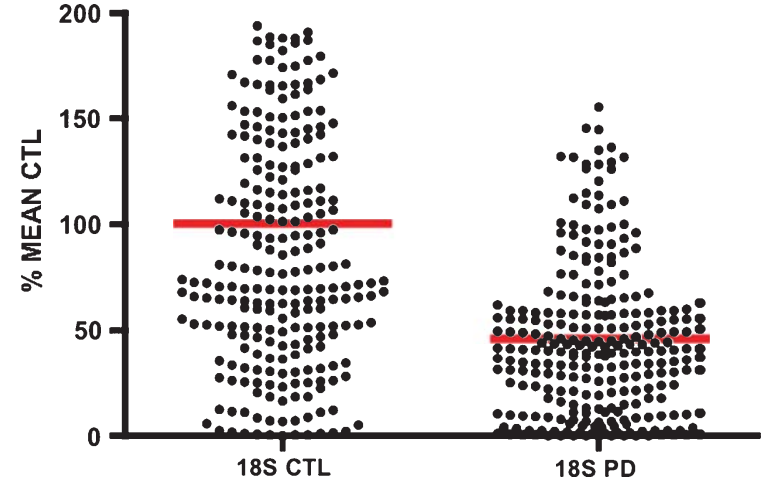

Fig. 1. Distribution of expression of nDNA-encoded Complex-I subunits in CTL and PD frontal cortex. Shown are expressions of all NDU subunits in 8 CTL and 8 PD samples normalized to expression of $18 \mathrm{~S}$ housekeeping gene in each sample. Populations were compared by Mann-Whitney test and are different at $p<0.0001$.

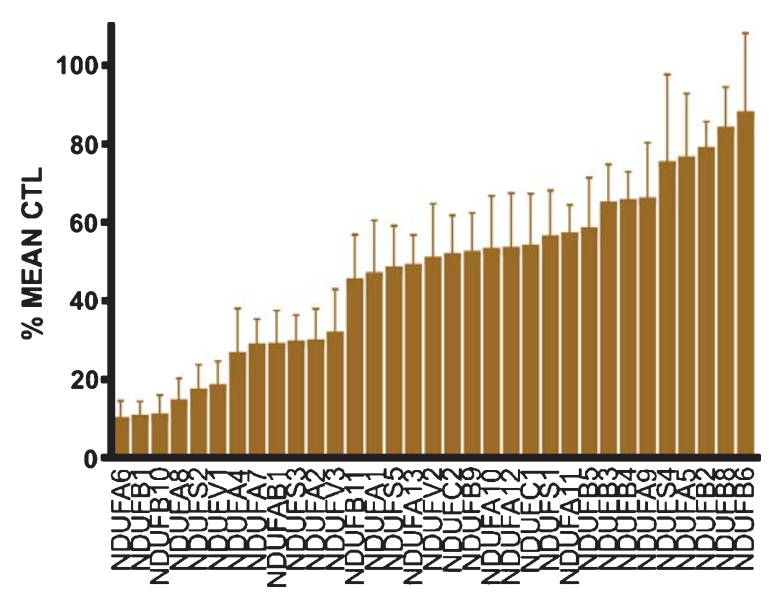

Fig. 2. Changes in nDNA-encoded NDU gene expression in PD compared to CTL brain samples. Shown are mean \pm SEM values for 8 PD samples where each was expressed as \% of mean CTL value. Two-way ANOVA across disease (PD vs. CTL) showed a highly significant effect of disease $(p<0.0001)$.

and stood out from changes in the expression levels of the other five known Complex-I assembly factors.

We then compared normalized expression levels of four Complex-I proteins (present study) to porinnormalized levels of the same Complex-I proteins in gradient-purified mitochondria reported in an earlier study [19]. Figure 4 shows that for NDUFB4 and NDUFA9, but not for NDUFS3 or NDUFB8, there were moderate correlations among gene expression levels and mitochondrial protein levels.

The reduction of multiple nDNA-encoded ComplexI genes could arise in part from reductions in mitobiogenesis signaling. Figure 5 presents results from analysis of expression of upstream genes (TFAM,

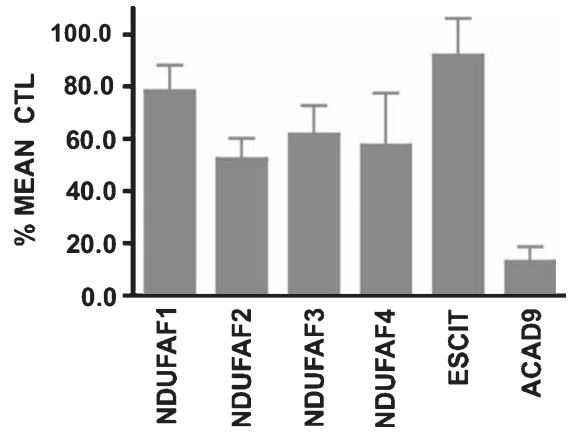

Fig. 3. Changes in gene expression for the six known ComplexI assembly factors. Shown are the mean \pm SEM values for gene expression of six known Complex-I assembly factors expressed as $\%$ of mean CTL value. ACAD9 expression in PD samples was significantly different from CTL ( $p=0.007$, Mann-Whitney test).
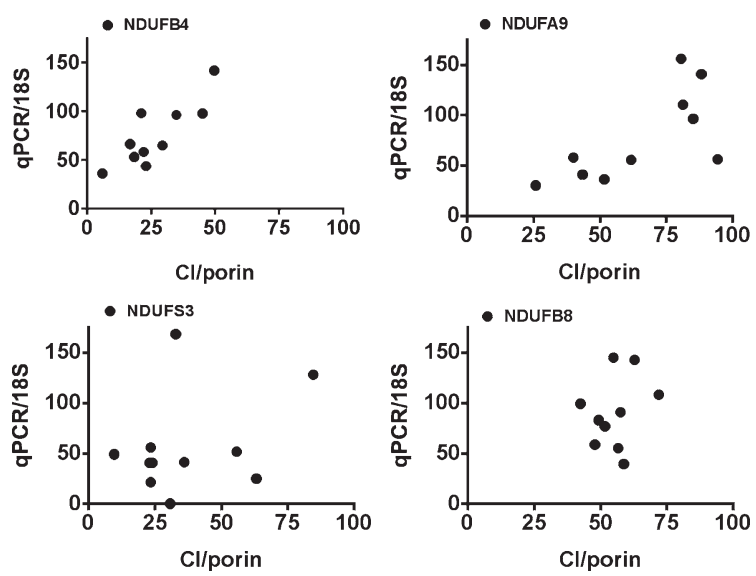

Fig. 4. Relationships among gene expressions of four nDNAencoded Complex-I proteins and their levels in gradient-purified mitochondria from frontal cortex samples. Frontal cortex, gradientpurified mitochondrial protein levels normalized to porin were determined as described in Arthur et al. [19] and used many of the same samples as in the present study. All values are presented as \% of mean CTL values for both PD $(n=7-8)$ and CTL $(n=4-5)$ samples.

TFB1M, PGC-1 $\alpha$, NRF1) that regulate mitochondrial biogenesis [17, 20, 21]. All showed a significant effect by two-way ANOVA of disease on expression in brain.

We were then curious as to whether mitobiogenesis signaling was preserved in PD, in terms of upstreamdownstream relationships. Figure 6 shows that the normalized expressions of the "downstream" mitobiogenesis genes nuclear respiratory factor-1 (NRF1) and mitochondrial transcription factor A (TFAM), but not that of mitochondrial transcription factor B1 (TFB1M), correlated well with that of the master mitobiogenesis "upstream" cofactor PGC- $1 \alpha$ in both PD and CTL samples. For NRF1 and TFAM, the correlation lines 


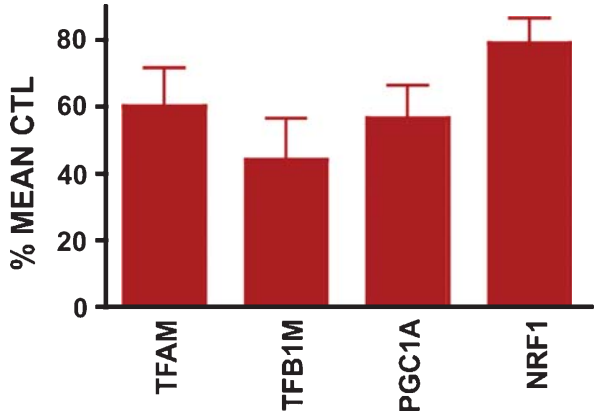

Fig. 5. Changes in gene expression for mitobiogenesis genes. Shown are the mean \pm SEM values for gene expression of mitobiogenesis genes in PD samples, expressed as \% of mean CTL value. Twoway ANOVA showed a significant effect of disease (CTL vs. PD, $p=0.0002$ ). Bonferroni testing showed that TFB1M was significantly different between CTL and PD $(P<0.05)$.
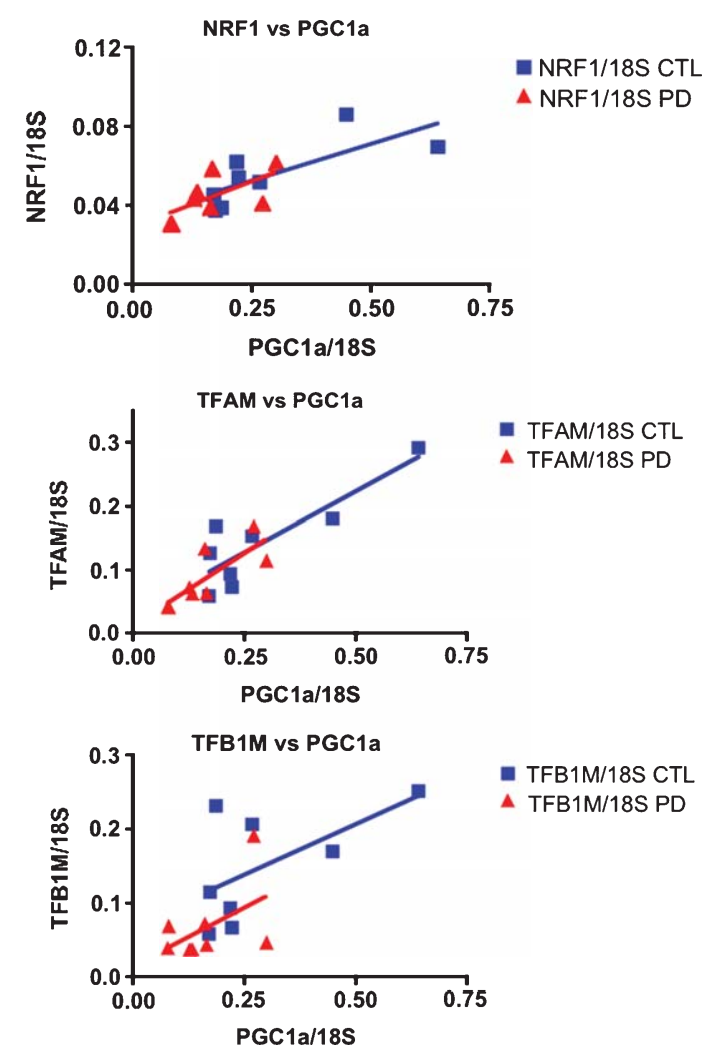

Fig. 6. Correlations among mitobiogenesis signaling genes. Shown are relationships in PD and CTL brain samples among expressions of PGC- $1 \alpha$ and NRF1 (top), TFAM (middle) and TFB1M (bottom). PD data are shown in red triangles, CTL data in blue squares.

against PGC1 $\alpha$ were almost identical in PD and CTL samples, suggesting similar biological control.

If expression of PGC- $1 \alpha$ and its downstream mitobiogenesis signaling factors relate to control of
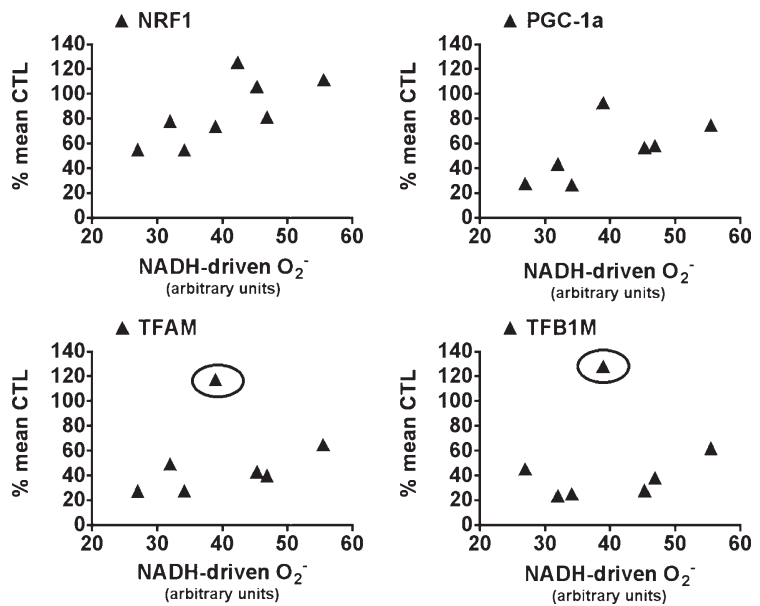

Fig. 7. Relationships among expression levels of mitobiogenesis transcription factors and mitochondrial NADH-driven electron transfer rates. Gradient-purified mitochondria and their NADHdriven superoxide production rates were measured in PD and CTL frontal cortex samples as described in Keeney et al. [18]. Shown are their relationships to normalized expression of mitobiogenesis transcription factors in the same samples used in the present study.

mitochondrial bioenergetics in our frontal cortical samples, then there should be relationships among the expressions of the mitobiogenesis factors and mitochondrial NADH-driven electron flow. Figure 7 shows that this appears variably to be the case for all the mitobiogenesis factors, if outlier values (circled) are not considered.

Figure 8 shows that expression of the Complex-I assembly factor ACAD9, which was reduced $>80 \%$ in PD samples, correlated variably in CTL but not PD samples with mitobiogenesis factors. This suggests that ACAD9 is not regulated in PD cortex by these factors in the manner that NRF1 and TFAM appear to be regulated by $\mathrm{PGC} 1 \alpha$ (Fig. 6).

We next examined expression patterns for the seven Complex-I genes ("ND") coded by mtDNA. Figure 9 shows that there was substantial heterogeneity among the PD samples in terms of levels of ND gene expression. Overall, relative expression levels were very similar within a sample, with over- or under-expression relative to mean CTL values across individual samples.

Because the relative expression levels of the seven mtDNA Complex-I "ND” genes within a given sample were so similar, we averaged them and sought to determine any relationships between the ND expression levels and those of mitochondrial biogenesis factors presented in Fig. 5. We found no apparent relationship except for NRF1, where as shown in Fig. 10 there 


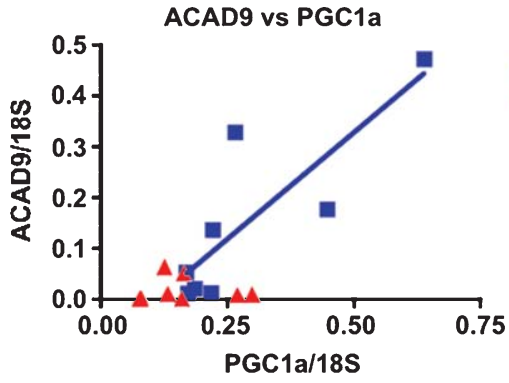

ACAD9/18S CTL

- ACAD9/18S PD

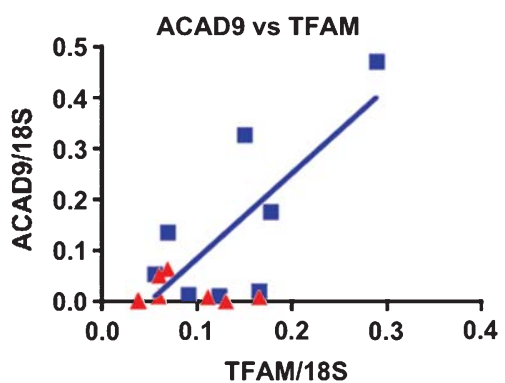

ACAD9/18S CTL $\triangle \mathrm{ACAD} 9 / 18 \mathrm{~S} P \mathrm{P}$

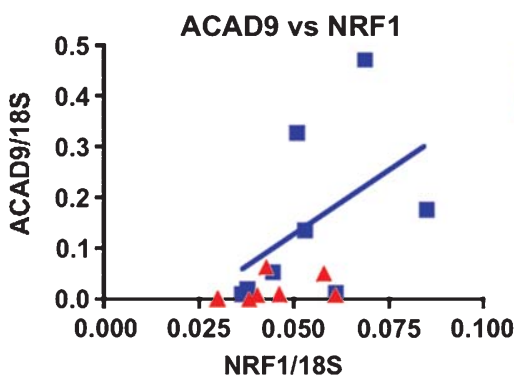

ACAD9/18S CTL $\triangle \mathrm{ACAD} 9 / 18 \mathrm{~S} P \mathrm{D}$

Fig. 8. Lack of correlation in PD and CTL samples among expression of the Complex-I assembly factor ACAD9 and PGC- $1 \alpha$, TFAM and NRF1. Shown are relationships in PD and CTL brain samples among expressions normalized to $18 \mathrm{~S}$ of ACAD9 and PGC- $1 \alpha$ (top), TFAM (middle) and NRF1 (bottom). PD data are shown in red triangles, CTL data in blue squares.

was a significant correlation among averaged ND gene expression levels in a given sample and that sample's relative expression level for NRF1.

We searched for changes in expression of the zincfinger protein ZNF 746, also known as "PARIS", that appears capable of regulating PGC-1 $\alpha$ expression [24]. We did not find any changes in PARIS expression in our PD brain samples $(\mathrm{CTL}=100 \pm 12 \% ; \mathrm{PD}=92 \pm 11 \%$ of mean CTL).

MicroRNA's are non-coding, small RNA molecules that can increase or decrease transcript stability and translation [25]. We assayed relative miRNA levels in our PD and CTL brain samples using Agilent miRNA arrays and Genespring XL to process results. We identified 48 miRNA's that were regulated $>2$-fold in the PD samples. Of these, microRNA.org (http:// cbio.mskcc.org/microrna-previous/getGeneForm.do)

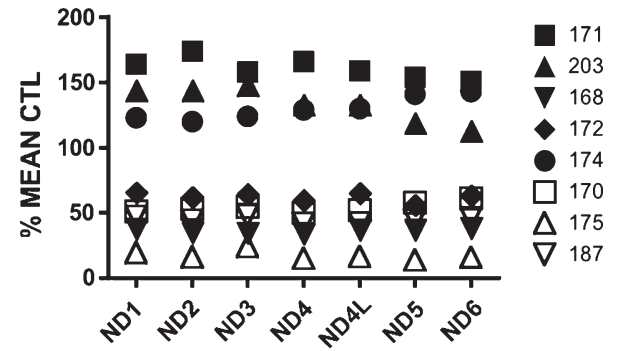

Fig. 9. Relative expression levels for mtDNA-encoded ComplexI genes in PD brain. Shown are the expression levels for the 7 mtDNA-encoded Complex-I genes in the individual PD brain samples, expressed as percent of mean CTL levels. Shown to the right are the code numbers for each sample.

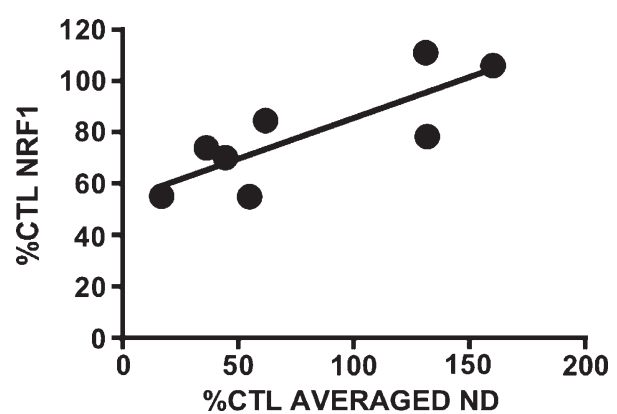

Fig. 10. Linear relationship among averaged ND gene expression levels in PD brain and expression levels for the mitobiogenesis factor NRF1. The values for expression of the seven ND genes in each sample were averaged and plotted against the expression values for NRF1 in each sample. The resulting plot had a $R^{2}$ value of 0.66 and $p=0.0138$.

Table 1

MicroRNA's altered in $8 \mathrm{PD}$ brains samples compared to $8 \mathrm{CTL}$ brain samples

\begin{tabular}{lcll}
\hline MicroRNA & $\begin{array}{c}\text { Fold- } \\
\text { change }\end{array}$ & Direction & $\begin{array}{l}\text { Potential } \\
\text { gene interactions }\end{array}$ \\
\hline hsa-miR-200b* & 4.16 & up & PGC1a, FOXO1, CREB5 \\
hsa-miR-200a* & 3.57 & up & PGC1a, FOXO1, CREB5 \\
hsa-miR-195* & 2.19 & up & FOXO1 \\
hsa-miR-424* & 2.18 & up & FOXO1 \\
& & & \\
hsa-miR-200a & 2.72 & down & PGC1a, FOXO1, CREB5 \\
hsa-miR-199a-3p & 2.65 & down & PGC-1a \\
hsa-miR-148a & 2.64 & down & MEF2, PGC1a \\
hsa-miR-451 & 2.45 & down & MEF2, ATF2 \\
hsa-miR-144 & 2.12 & down & MEF2, FOXO1, PGC1a \\
hsa-miR-429 & 2.05 & down & PGC1a, FOXO1, CREB5 \\
hsa-miR-190 & 2.02 & down & CREB5, MEF2 \\
\hline
\end{tabular}

predicted 11 miRNA families that could interact with PGC-1 $\alpha$, or its upstream regulators (MEF2, FOXO1, ATF2, CREBx) (Table 1). 


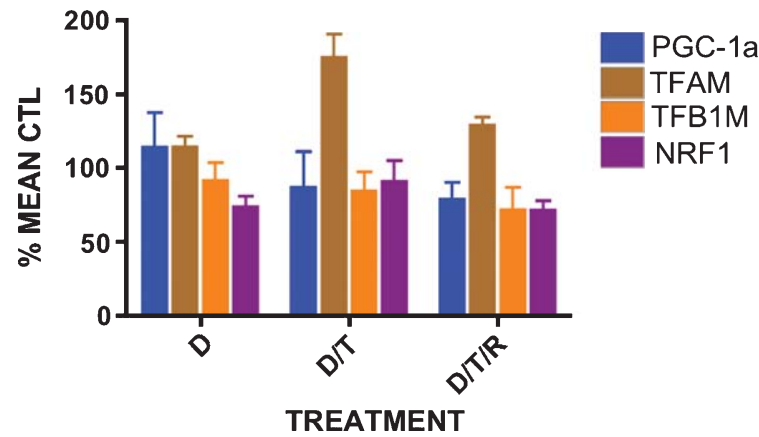

Fig. 11. Effects of one week's treatment of differentiated DAN neurons with DETA-NO alone ("D"), or combined with TNF- $\alpha$ ("D/T") or rotenone ("D/T/R") on expression of mitobiogenesis genes. Shown are the means $+/-$ SEM of three independent experiments. The treatments caused $\sim 50 \%$ loss of cells.

We then attempted to mimic the stressful environment, which PD brain cells might experience [26, 27], to determine if such in vitro conditions altered mitobiogenesis signaling. We used human DAN neurons grown in differentiation media (supplemental Figure 1) and exposed to the long acting NO generator DETANO, or DETA-NO and the cytokine TNF-alpha, or DETA-NO, TNF-alpha and the Complex-I inhibitor rotenone. As shown in Fig. 11, one week of these exposures tended to up regulate TFAM but down regulate PGC-1 $\alpha$, TFB1M and NRF1. These exposures produced $\sim 50 \%$ loss of cells in one week (not shown).

\section{DISCUSSION}

Our findings add to the accumulating evidence that PD brains experience a paradoxical "divorcing" of their mitochondria, manifested by widespread down regulation of energy producing genes in multiple sites with the brain, in brain tissue from those not yet with clinical PD but likely to develop it, and in peripheral PD tissues [16]. Many of the under-expressed genes found in PD tissues are regulated by the master mitobiogenesis coactivator PGC- $1 \alpha[16,17,20,21,28]$. We found in our PD brain samples that linear correlations between expression of PGC- $1 \alpha$ and its downstream targets NRF1 and TFAM were preserved $\sim$ identically to those of CTL brains. This suggests that the problem in PD brain does not lie with mitobiogenesis signaling downstream of PGC- $1 \alpha$, which appears preserved, rather that the upstream signaling is impaired.

A recent discovery in this area is the parkininteracting, zinc-finger protein ZNF746 (PARIS) that regulates nigral neuron PGC-1 $\alpha$ expression [24]. We did not find PARIS expression to change in our PD brains in this study, indicating that dysregulation of PARIS expression is not likely to be the explanation of impaired mitobiogenesis in our samples.

Whatever the mechanisms are for impaired mitobiogenesis signaling in our frontal cortical samples, mitobiogenesis signaling factor expression appeared to predict mitochondrial respiratory electron transfer rates as reflected by NADH-driven superoxide production [18]. This observation defines the existence of a fundamental relationship between mitobiogenesis signaling factor expression and a reduction of physiological (NADH-driven) mitochondrial electron transfer function in PD frontal cortex.

Complex-I is the largest multi-protein Complex in the respirasome [29] and is responsible for oxidizing the eight molecules of NADH formed from by oxidative decarboxylation of glucose-derived pyruvates in the tricarboxylic acid cycle. In PD frontal cortex samples, we found that Complex-I is oxidatively damaged and has reduced NADH-driven electron flow [18]. This reduced NADH-driven electron flow correlated with the level of oxidative damage to Complex-I proteins, implying a causal relationship [18].

In the same PD frontal cortex samples used in the present study, levels of multiple Complex-I protein subunits in gradient purified mitochondria were also reduced [19]. Because these Complex-I protein subunit levels were normalized to porin, this implies that either their synthesis was impaired, their incorporation into respirasomes was reduced, their degradation was increased, or some combination of processes occurred. We found that two (NDUFB4, NDUFA9) out of the four genetically defined nDNA-encoded Complex-I proteins reduced in mitochondria isolated from our PD frontal cortical samples [19] appear to be primarily transcriptionally regulated. It is not known to us how the other two Complex-I proteins (NDUFS3, NDUFB8) are primarily regulated, but the mechanisms appear to be post-transcriptional.

Complex-I assembly is also regulated by at least six known assembly factors, including the recently described ACAD9, a member of the acyl-CoA dehydrogenase family [23]. ACAD9 closely resembles very long-chain acyl-CoA dehydrogenase (VLCAD) that is involved in mitochondrial beta-oxidation of longchain fatty acids. ACAD9 binds to the previously recognized Complex-I assembly factors NDUFAF1 and Escit, which showed much less reduction in our PD samples [23]. It is not clear why ACAD9 expression was so markedly reduced $(\sim 87 \%)$ in our samples, 
but this finding suggests that PD brain Complex-I may experience mis-assembly as a result of ACAD9 deficiency, in addition to reduced expression of multiple nDNA-encoded Complex-I subunits. We did not find any evidence that ACAD9 expression was related to mitobiogenesis gene signaling, implying that it is separately regulated.

PD brains have been shown to experience increased nitrosative and oxidative stresses $[18,30]$ and demonstrate microglial activation with increased levels of apoptosis-inducing cytokines, including TNF- $\alpha$ [26]. We attempted to mimic the environment of nitrosative stress, oxidative stress and elevated cytokines by incubating differentiated human DAN neurons with NO, \pm TNF-alpha, \pm rotenone for 3 days. We reproduced some (NRF1,TFB1M, PGC-1 $\alpha$ ), but importantly not all (TFAM) of the trends for change in expression of mitobiogenesis factors found in PD brain. This suggests that the toxic environment observed in PD brain could contribute to impaired mitobiogenesis signaling but is not likely to be the complete explanation.

In summary, our findings in PD frontal cortex samples support and extend the earlier findings that PD subjects appear to have a widespread brain and systemic down-regulation of mitochondrial energy production [16]. We found apparent preservation of upstream-downstream mitobiogenesis signaling relationships, but a widespread decrease in expression of multiple nDNA-encoded Complex-I genes. Both the changes in mitobiogenesis factors' expressions and nDNA-encoded Complex-I gene expressions appeared to have functional correlates with NADH-mediated electron flow and some mitochondrial protein levels, respectively. We found a completely different regulation pattern of the $7 \mathrm{mtDNA}$-encoded Complex-I genes that are believed to be involved mainly in proton pumping $[31,32]$. Our findings imply that these two gene families are independently regulated in PD brain and linked together by expression of upstream mitobiogenesis factors [17, 20, 21].

The origins of these important bioenergetic changes in PD brain are likely multifactorial. Our initial experiments suggest that local environmental changes and microRNA expression alterations are potentially involved. If true, the relative proportion of involvement in individual cases may be heterogeneous. We are intrigued by the finding that microRNA regulation of upstream regulators of PGC- $1 \alpha$ expression may contribute to pathogenesis of our observed Complex-I subunit expression changes.

We also do not know if markers for impaired mitobiogenesis could represent reliable biomarkers for PD development. We are exploring the possibility that peripheral cell expression of mitobiogenesis and mitochondrial bioenergetic genes, along with altered microRNA levels, might be helpful in that regard.

Finally, our findings and those of others support the approach that stimulation of mitochondrial biogenesis could alter loss of bioenergetic activity in PD. Such an approach is now available with recombinant human TFAM that has consistently stimulated mitobiogenesis in a variety of cell models and in vivo in mice [33-37]. If frontal cortex mitobiogenesis can be stimulated in $\mathrm{PD}$, appearance and progression of disabling cognitive and behavioral deficits in this disease might be slowed.

\section{COMPETING INTERESTS}

The authors declare no competing interests with respect to the content of this manuscript. JPB is the inventor on patents protecting the use of $\mathrm{R}(+)$ pramipexole in neurodegenerative diseases, including $\mathrm{PD}$, and receives royalty payments. $\mathrm{R}(+)$ pramipexole is a mitochondrially active drug but is not discussed in this paper.

\section{AUTHOR'S CONTRIBUTIONS}

RRT prepared cDNA's from brain tissues and carried out all qPCR experiments and data analyses in brain samples. He was consistently blinded as to sample identity (PD vs. CTL). PMK prepared RNA extracts of brain samples, cultured DAN cells and carried out all cell experiments including qPCR studies on cell gDNA and cDNA. JPB designed experiments, analyzed all unblinded data including those from miRNA arrays and wrote the manuscript.

\section{ACKNOWLEDGMENTS}

This work was supported by NIH grants NS39788 and the VCU Parkinson's Disease Center (www.parkinsons.vcu.edu). 


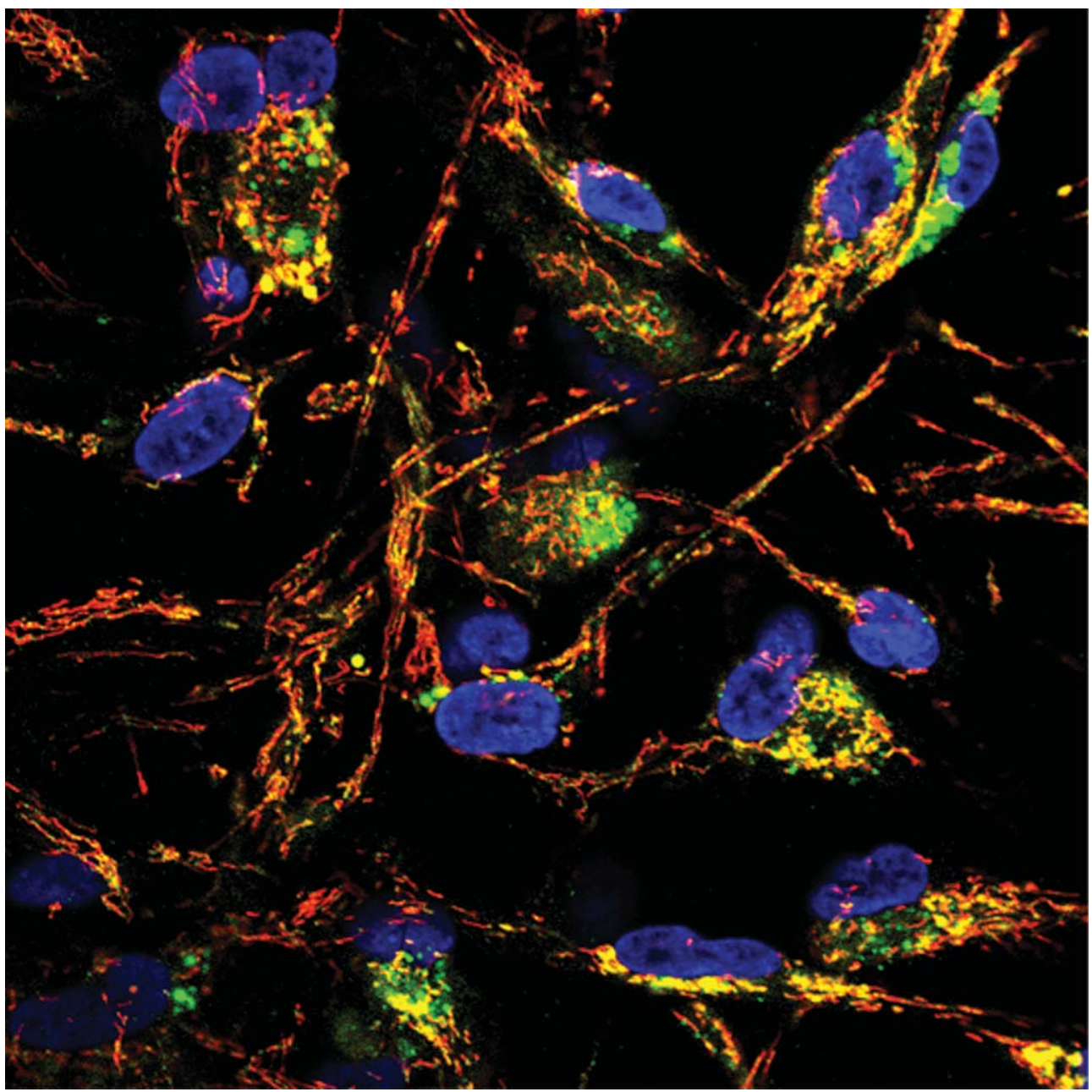

Supplemental Figure 1. Human DAN neurons in culture, immunostained for Complex-I (green), counterstained with MitoTracker Red and DAPI (blue).

\section{REFERENCES}

[1] Bertrand E, Lechowicz W, Szpak GM, Lewandowska E, Dymecki J, \& Wierzba-Bobrowicz T (2004) Limbic neuropathology in idiopathic Parkinson's disease with concomitant dementia. Folia Neuropathol, 42, 141-150.

[2] Braak E, Sandmann-Keil D, Rub U, Gai WP, de Vos RA, Steur EN, Arai K, \& Braak H (2001) alpha-synuclein immunopositive Parkinson's disease-related inclusion bodies in lower brain stem nuclei. Acta Neuropathol (Berl), 101, 195-201.

[3] Braak H, de Vos RA, Bohl J, \& Del Tredici K (2006) Gastric alpha-synuclein immunoreactive inclusions in Meissner's and Auerbach's plexuses in cases staged for Parkinson's diseaserelated brain pathology. Neurosci Lett, 396, 67-72.

[4] Braak H, Del Tredici K, Rub U, de Vos RA, Jansen Steur EN, \& Braak E (2003) Staging of brain pathology related to sporadic Parkinson's disease. Neurobiol Aging, 24, 197-211.

[5] Braak H, Ghebremedhin E, Rub U, Bratzke H, \& Del Tredici K (2004) Stages in the development of Parkinson's diseaserelated pathology. Cell Tissue Res, 318, 121-134.
[6] Braak H, Sandmann-Keil D, Gai W, \& Braak E (1999) Extensive axonal Lewy neurites in Parkinson's disease: A novel pathological feature revealed by alpha-synuclein immunocytochemistry. Neurosci Lett, 265, 67-69.

[7] Del Tredici K, Rub U, De Vos RA, Bohl JR, \& Braak H (2002) Where does parkinson disease pathology begin in the brain? J Neuropathol Exp Neurol, 61, 413-426.

[8] Chaudhuri KR, Odin P, Antonini A, \& Martinez-Martin P (2011) Parkinson's disease: The non-motor issues. Parkinsonism Relat Disord, 17, 717-723.

[9] Chaudhuri KR, \& Schapira AH (2009) Non-motor symptoms of Parkinson's disease: Dopaminergic pathophysiology and treatment. Lancet Neurol, 8, 464-474.

[10] Belin AC, \& Westerlund M (2008) Parkinson's disease: A genetic perspective. FEBS J, 275, 1377-1383.

[11] Lesage S, \& Brice A (2009) Parkinson's disease: From monogenic forms to genetic susceptibility factors. Hum Mol Genet, 18, R48-R59.

[12] Bardien S, Lesage S, Brice A, \& Carr J (2011) Genetic characteristics of leucine-rich repeat kinase 2 (LRRK2) associated 
Parkinson's disease. Parkinsonism Relat Disord, 17, 501-508.

[13] Burbulla LF, \& Kruger R (2011) Converging environmental and genetic pathways in the pathogenesis of Parkinson's disease. J Neurol Sci, 306, 1-8.

[14] Sato S, \& Hattori N (2011) Genetic mutations and mitochondrial toxins shed new light on the pathogenesis of Parkinson's disease. Parkinsons Dis, 2011, 979231.

[15] Yue Z, \& Lachenmayer ML (2011) Genetic LRRK2 models of Parkinson's disease: Dissecting the pathogenic pathway and exploring clinical applications. Mov Disord, 26, 1386-1397.

[16] Zheng B, Liao Z, Locascio JJ, Lesniak KA, Roderick SS, Watt ML, Eklund AC, Zhang-James Y, Kim PD, Hauser MA, Grunblatt E, Moran LB, Mandel SA, Riederer P, Miller RM, Federoff HJ, Wullner U, Papapetropoulos S, Youdim MB, Cantuti-Castelvetri I, Young AB, Vance JM, Davis RL, Hedreen JC, Adler CH, Beach TG, Graeber MB, Middleton FA, Rochet JC, \& Scherzer CR (2010) PGC-1alpha, a potential therapeutic target for early intervention in Parkinson's disease. Sci Transl Med, 2, 52ra73.

[17] Scarpulla RC (2011) Metabolic control of mitochondrial biogenesis through the PGC-1 family regulatory network. Biochim Biophys Acta, 1813, 1269-1278.

[18] Keeney PM, Xie J, Capaldi RA, \& Bennett JP Jr (2006) Parkinson's disease brain mitochondrial complex I has oxidatively damaged subunits and is functionally impaired and misassembled. J Neurosci, 26, 5256-5264.

[19] Arthur CR, Morton SL, Dunham LD, Keeney PM, \& Bennett JP Jr (2009) Parkinson's disease brain mitochondria have impaired respirasome assembly, age-related increases in distribution of oxidative damage to mtDNA and no differences in heteroplasmic mtDNA mutation abundance. Mol Neurodegener, $\mathbf{4}, 37$.

[20] Scarpulla RC (2006) Nuclear control of respiratory gene expression in mammalian cells. $J$ Cell Biochem, 97, 673-683.

[21] Scarpulla RC (2008) Transcriptional paradigms in mammalian mitochondrial biogenesis and function. Physiol Rev, 88, 611-638.

[22] Borland MK, Mohanakumar KP, Rubinstein JD, Keeney PM, Xie J, Capaldi R, Dunham LD, Trimmer PA, \& Bennett JP Jr (2009) Relationships among molecular genetic and respiratory properties of Parkinson's disease cybrid cells show similarities to Parkinson's brain tissues. Biochim Biophys Acta, 1792, 68-74.

[23] Nouws J, Nijtmans L, Houten SM, van den Brand M, Huynen M, Venselaar H, Hoefs S, Gloerich J, Kronick J, Hutchin T, Willems P, Rodenburg R, Wanders R, van den Heuvel L, Smeitink J, \& Vogel RO (2010) Acyl-CoA dehydrogenase 9 is required for the biogenesis of oxidative phosphorylation complex I. Cell Metab, 12, 283-294.

[24] Shin JH, Ko HS, Kang H, Lee Y, Lee YI, Pletinkova O, Troconso JC, Dawson VL, \& Dawson TM (2011) PARIS (ZNF746) repression of PGC-1alpha contributes to neurodegeneration in Parkinson's disease. Cell, 144, 689-702.

[25] Junn E, \& Mouradian MM (2012) MicroRNAs in neurodegenerative diseases and their therapeutic potential. Pharmacol Ther, 133, 142-150.
[26] Nagatsu T (2002) Parkinson's disease: Changes in apoptosisrelated factors suggesting possible gene therapy. J Neural Transm, 109, 731-745.

[27] Yokoyama H, Uchida H, Kuroiwa H, Kasahara J, \& Araki $\mathrm{T}$ (2011) Role of glial cells in neurotoxin-induced animal models of Parkinson's disease. Neurol Sci, 32, 1-7.

[28] Zheng B, Liao Z, Locascio JJ, Lesniak KA, Roderick SS, Watt ML, Eklund AC, Zhang-James Y, Kim PD, Hauser MA, Grunblatt E, Moran LB, Mandel SA, Riederer P, Miller RM, Federoff HJ, Wullner U, Papapetropoulos S, Youdim MB, Cantuti-Castelvetri I, Young AB, Vance JM, Davis RL, Hedreen JC, Adler CH, Beach TG, Graeber MB, Middleton FA, Rochet JC, \& Scherzer CR (2010) PGC-1alpha, a potential therapeutic target for early intervention in Parkinson's disease. Sci Transl Med, 2, 52ra73.

[29] Acin-Perez R, Fernandez-Silva P, Peleato ML, Perez-Martos A, \& Enriquez JA (2008) Respiratory active mitochondrial supercomplexes. Mol Cell, 32, 529-539.

[30] Moore DJ, West AB, Dawson VL, \& Dawson TM (2005) Molecular pathophysiology of Parkinson's disease. Annu Rev Neurosci, 28, 57-87.

[31] Drose S, Krack S, Sokolova L, Zwicker K, Barth HD, Morgner N, Heide H, Steger M, Nubel E, Zickermann V, Kerscher S, Brutschy B, Radermacher M, \& Brandt U (2011) Functional dissection of the proton pumping modules of mitochondrial complex I. PLoS Biol, 9, e1001128.

[32] Hunte C, Zickermann V, \& Brandt U (2010) Functional modules and structural basis of conformational coupling in mitochondrial complex I. Science, 329, 448-451.

[33] Iyer S, Thomas RR, Portell FR, Dunham LD, Quigley CK, \& Bennett JP Jr (2009) Recombinant mitochondrial transcription factor $\mathrm{A}$ with $\mathrm{N}$-terminal mitochondrial transduction domain increases respiration and mitochondrial gene expression. Mitochondrion, 9, 196-203.

[34] Iyer S, Xiao E, Alsayegh K, Eroshenko N, Riggs MJ, Bennett JP Jr, \& Rao RR (2011) Mitochondrial gene replacement in human pluripotent stem cell-derived neural progenitors. Gene Ther.

[35] Keeney PM, Quigley CK, Dunham LD, Papageorge CM, Iyer S, Thomas RR, Schwarz KM, Trimmer PA, Khan SM, Portell FR, Bergquist KE, \& Bennett JP (2009) Mitochondrial Gene Therapy Augments Mitochondrial Physiology in a Parkinson's Disease Cell Model. Hum Gene Ther, 20, 897-907.

[36] Khan SM, \& Bennett JP Jr (2004) Development of mitochondrial gene replacement therapy. J Bioenerg Biomembr, 36, 387-393.

[37] Thomas RR, Khan SM, Portell FR, Smigrodzki RM, \& Bennett JP Jr (2011) Recombinant human mitochondrial transcription factor A stimulates mitochondrial biogenesis and ATP synthesis, improves motor function after MPTP, reduces oxidative stress and increases survival after endotoxin. Mitochondrion, 11, 108-118. 